\title{
Treatment of unusual locked posterior fracture-dislocation of the shoulder: a case series
}

\author{
Ho Yeon Park, Seok Jung Kim, Yoo Joon Sur, Jae Woong Jung, Chae-gwan Kong \\ Department of Orthopedic Surgery, College of Medicine, The Catholic University of Korea, Seoul, Korea
}

Background: Locked posterior fracture-dislocation of the shoulder (LPFDS) is a very rare injury that occurs predominantly in young patients following high-energy trauma. The long-term outcome of the treatment of this injury is often poor. This study sought to present the characteristics of injury, discuss the pathological anatomy, and to report the treatment outcomes of our case series.

Methods: Between January 2012 and May 2018, a total of 234 patients who underwent surgical treatment for proximal humerus fractures were reviewed. Among them, six patients (mean age, 54.7 years; range, 35-76 years) with LPFDS were included in this study. Four patients were treated with open reduction and internal fixation (ORIF) with locking plates, one with hemiarthroplasty, and one with reverse total shoulder arthroplasty. Clinical results were evaluated by Constant, American Shoulder and Elbow Surgeons (ASES), and visual analog scale (VAS) scores and radiologic evaluation was conducted using follow-up radiographs.

Results: The mean length of follow-up was 26.2 months (range, 12-54). The mean Constant, ASES, and VAS scores were 66.7, 65.5, and 2.2, respectively. Four patients who underwent ORIF achieved bony union, but avascular necrosis (AVN) of the humeral head was observed in two patients. No complications were observed in the patients who underwent arthroplasty surgery until final follow-up.

Conclusions: In the treatment of LPFDS, replacement arthroplasty can produce predictable results. The approach of ORIF may be considered as a first choice of treatment in young patients but is sometimes correlated with postoperative complications such as AVN and the functional outcomes may be unpredictable. Therefore, patients should undergo careful diagnosis and treatment of this type of injury.

Keywords: Shoulder; Humerus; Fracture dislocation; Posterior

\section{INTRODUCTION}

Dislocation of the shoulder is a common injury, with most cases being anterior dislocation. Posterior dislocation of the shoulder (PDS) is a rare injury accounting for only $2 \%$ to $5 \%$ of all shoulder dislocations in the literature [1]. Meanwhile, posterior fracture-dislocation of the shoulder affected $0.9 \%$ of 1,500 cases re- ported by Neer, which is rarer [2,3]. The mechanisms of PDS can be classified as atraumatic and traumatic. The latter is mostly caused by high-energy trauma, while fracture is usually caused by axial loading, with the arm in an adducted, flexed, and internally rotated position [4].

In PDS, impacted articular fracture of the humeral head (reverse Hills-Sachs lesion) (Fig. 1) was the most common associat-

Received: August 14, 2020 Revised: August 28, $2020 \quad$ Accepted: August 28, 2020

Correspondence to: Chae-gwan Kong

Department of Orthopedic Surgery, Uijeongbu St. Mary's Hospital, College of Medicine, The Catholic University of Korea, 271 Cheonbo-ro, Uijeongbu 11765 , Korea

Tel: +82-31-820-3947, Fax: +82-31-847-3671, E-mail: gongjae@ catholic.ac.kr, ORCID: https://orcid.org/0000-0002-1956-3861

Financial support: None.

Conflict of interest: None.

Copyright@ 2020 Korean Shoulder and Elbow Society. All Rights Reserved.

This is an Open Access article distributed under the terms of the Creative Commons Attribution Non-Commercial License (http://creativecommons.org/licenses/by-nc/4.0/) which permits unrestricted non-commercial use, distribution, and reproduction in any medium, provided the original work is properly cited. 
ed feature, followed by humeral neck fractures, lesser tuberosity fracture, and greater tuberosity fracture [5]. In some cases, the lesion is complicated by a proximal humeral fracture, usually at the level of the anatomical neck. Some authors defined this injury as a locked posterior fracture-dislocation of the shoulder (LPFDS) or complex posterior fracture-dislocation of the shoulder (Fig. 2) $[6,7]$.

Since LPFDS is not commonly seen in daily clinical practice, early diagnosis is often missed [6] and there is no gold-standard treatment for LPFDS. Existing treatments vary from closed reduction and pinning to open reduction and internal fixation (ORIF) to replacement arthroplasty [8]. Arthroplasty has often been preferred over ORIF, especially among elderly patients because the results are predictable and severe complications such as nonunion or avascular necrosis (AVN) of the humeral head can

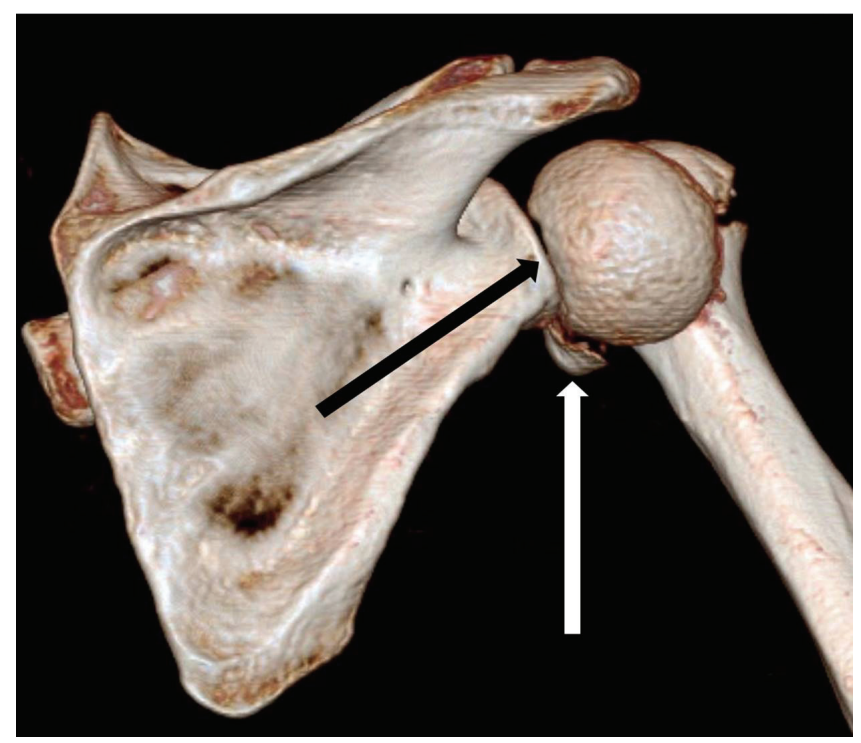

Fig. 1. A three-dimensional computed tomography scan of the shoulder shows an impacted articular fracture of the humeral head known as a reverse Hill-Sachs lesion (black arrow) and a fracture fragment (white arrow). occur after ORIF. However, according to recent studies, these complication rates have decreased as compared with previous reports [8]. The results of successful osteosynthesis surgery were superior to joint-replacement surgery; therefore, ORIF should be considered as a first-choice treatment in young patients with this type of injury. The authors of the present report experienced six cases with this rare type of injury in combination with a Neer four-part fracture. This study aimed to present the characteristics of injury, discuss the pathological anatomy, and report the treatment outcomes of our case series.

\section{METHODS}

This study was approved by the Institutional Review Board of Uijeongbu St. Mary's Hospital (IRB No. UC20RAS10120). Owing to the retrospective design, the requirement for informed consent was waived.

The medical records of 234 patients who underwent surgical treatment for proximal humerus fracture between January 2012 and May 2018 were reviewed retrospectively. During this period, patients who had undergone surgery for LPFDS and participated in follow-up for more than 6 months were included in this study. Six patients were identified and included in the study. The mean age was 54.7 years (range, 35-76 years) and three patients each of six total were male and female, respectively. The injury mechanisms included atraumatic seizure $(n=1)$, motorcycle accident $(\mathrm{n}=3)$, car accident $(\mathrm{n}=1)$, and a fall from a 3 -m height $(\mathrm{n}=1)$, respectively (Table 1 ).

One orthopedic shoulder surgeon (CGK) performed all operations. Under general anesthesia, a deltopectoral approach was used in all patients. ORIF using a PHILOS plate (DePuy Synthes, Oberdorf, Switzerland) was performed in four patients (Figs. 2-5), hemiarthroplasty (Aequalis; Tornier, Minneapolis, $\mathrm{MN}$, USA) in one patient (Fig. 6), and reverse total shoulder arthroplasty (Aequalis, Tornier) in one patient (Fig. 7). The clinical re-
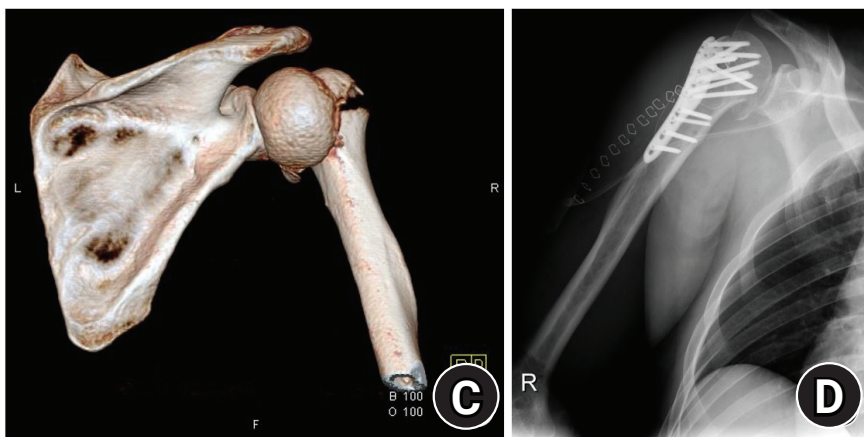

Fig. 2. Imaging of patient 1: preoperative X-ray (A), pre- and postoperative three-dimensional computed tomography scans (B, C), and postoperative X-ray (D). 
Table 1. Characteristics of the patients, fracture type, surgery methods, and results at the final follow-up visit

\begin{tabular}{|c|c|c|c|c|c|c|c|c|c|c|c|c|}
\hline $\begin{array}{l}\text { Patient } \\
\text { no. }\end{array}$ & Sex & $\begin{array}{l}\text { Age } \\
\text { (yr) }\end{array}$ & Side & $\begin{array}{c}\text { Neer } \\
\text { classification }\end{array}$ & $\begin{array}{c}\text { Mechanism } \\
\text { of injury }\end{array}$ & $\begin{array}{l}\text { Time of surgery } \\
\text { from the injury }\end{array}$ & Surgery & $\begin{array}{l}\text { Follow-up } \\
\text { (mo) }\end{array}$ & Complications & $\begin{array}{l}\text { VAS } \\
\text { score }\end{array}$ & $\begin{array}{c}\text { Constant } \\
\text { score }\end{array}$ & $\begin{array}{l}\text { ASES } \\
\text { score }\end{array}$ \\
\hline 1 & M & 35 & Right & 4 & Fall from height & $9 \mathrm{H}$ & ORIF & 54 & AVN & 3 & 44 & 39 \\
\hline 2 & $\mathrm{~F}$ & 43 & Right & 4 & Car accident & $26 \mathrm{H}$ & ORIF & 13 & & 1 & 88 & 87 \\
\hline 3 & M & 47 & Right & 4 & Motorbike & $16 \mathrm{H}$ & ORIF & 27 & AVN & 4 & 45 & 42 \\
\hline 4 & $\mathrm{M}$ & 51 & Right & 4 & Motorbike & $18 \mathrm{H}$ & ORIF & 16 & & 0 & 93 & 92 \\
\hline 5 & M & 76 & Right & 4 & Motorbike & $5 \mathrm{D}$ & RTSA & 12 & & 2 & 72 & 69 \\
\hline 6 & $\mathrm{~F}$ & 76 & Left & 4 & Seizure & $3 \mathrm{D}$ & Hemiarthroplasty & 35 & & 3 & 58 & 64 \\
\hline
\end{tabular}

VAS: visual analog scale, ASES: American Shoulder and Elbow Surgeons, ORIF: open reduction and internal fixation, AVN: avascular necrosis, RTSA: reverse total-shoulder arthroplasty.
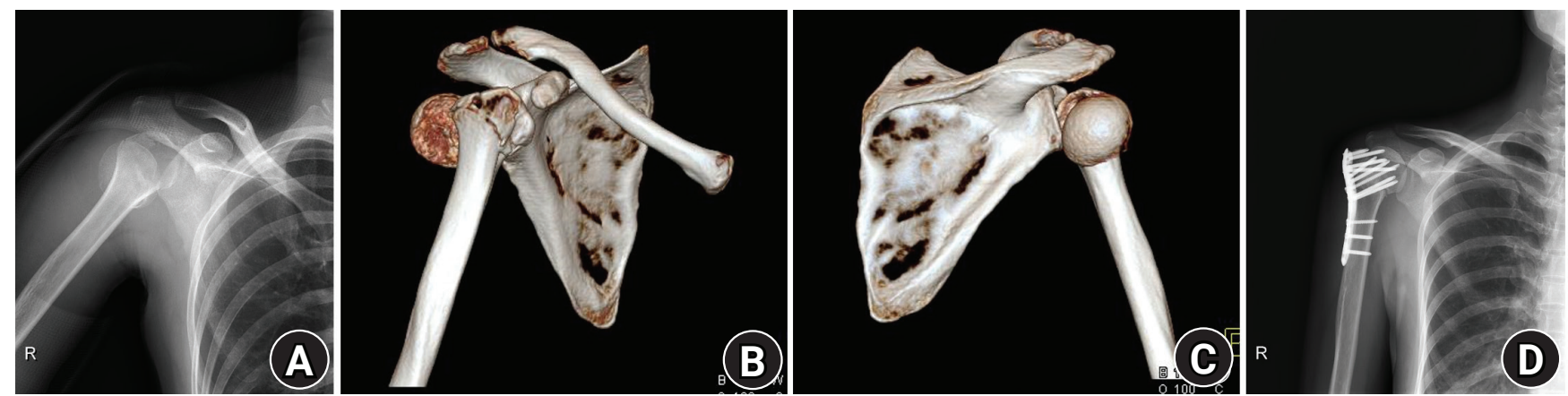

Fig. 3. Imaging of patient 2: preoperative X-ray (A), pre- and postoperative three-dimensional computed tomography scans (B, C), and postoperative X-ray (D).
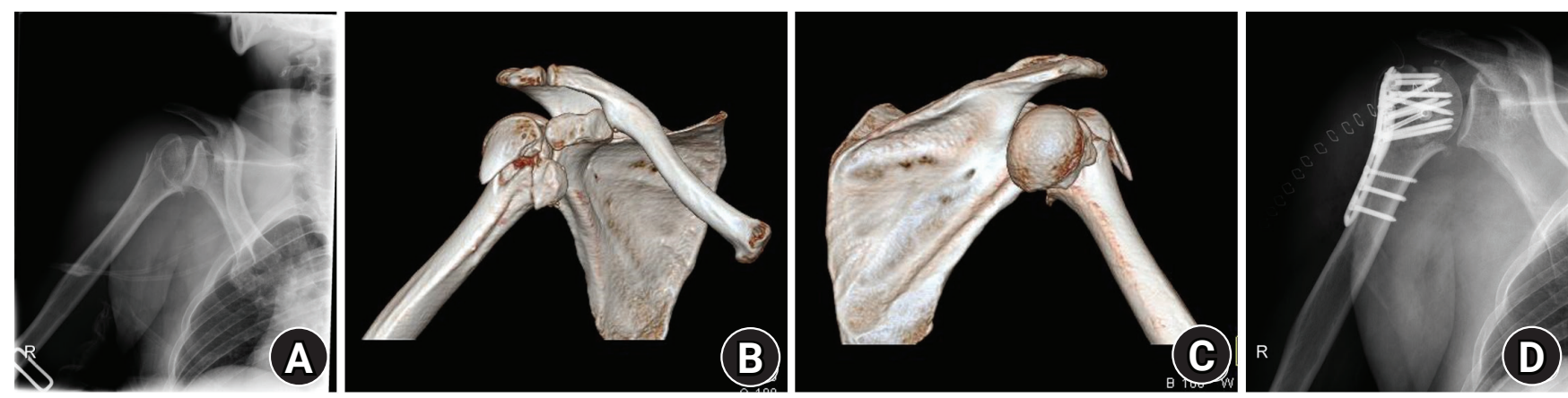

Fig. 4. Imaging of patient 3: preoperative X-ray (A), pre- and postoperative three-dimensional computed tomography scans (B, C), and postoperative X-ray (D).
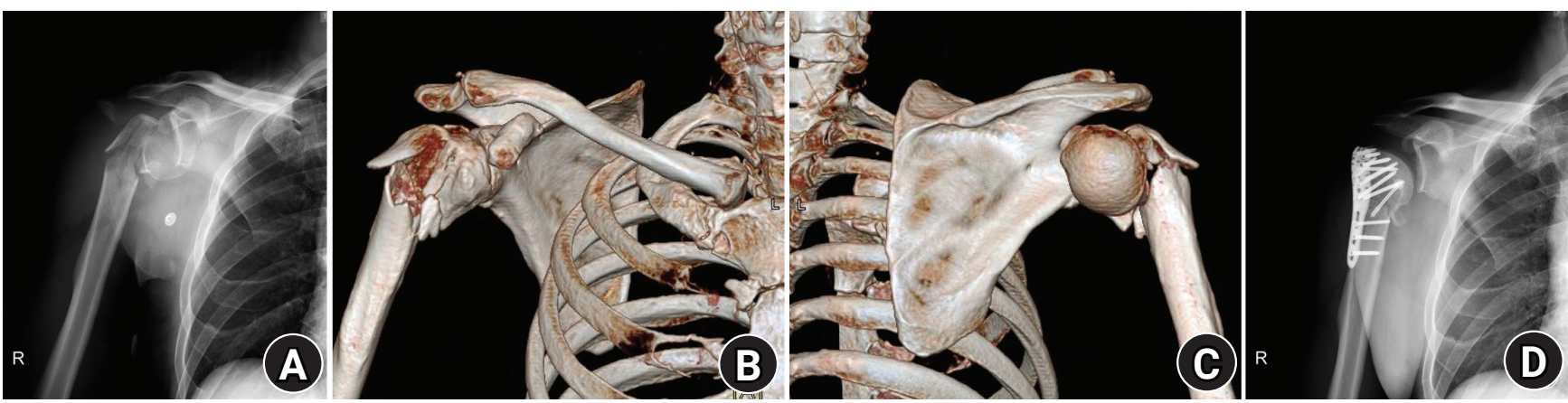

Fig. 5. Imaging of patient 4: preoperative X-ray (A), pre- and postoperative three-dimensional computed tomography scans (B, C), and postoperative X-ray (D). 

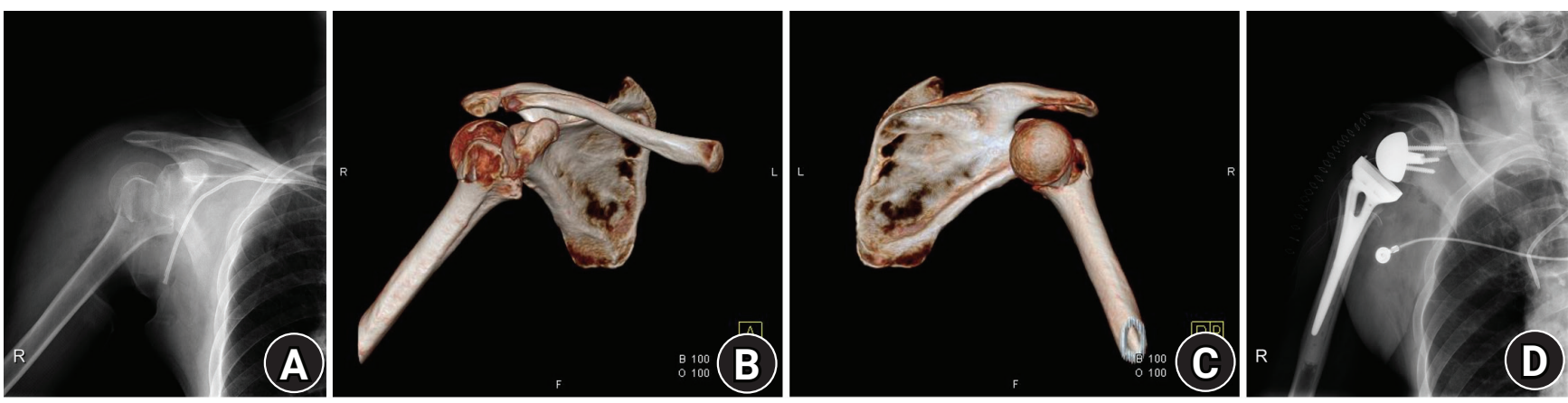

Fig. 6. Imaging of patient 5: preoperative X-ray (A), pre- and postoperative three-dimensional computed tomography scans (B, C), and postoperative X-ray (D).
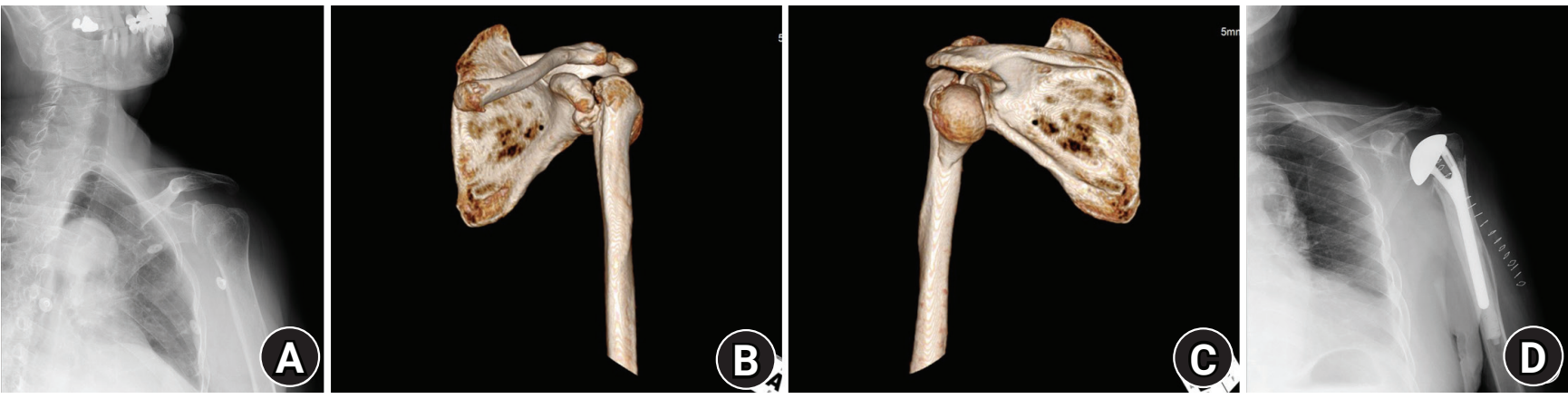

Fig. 7. Imaging of patient 6: preoperative X-ray (A), pre- and postoperative three-dimensional computed tomography scans (B, C), and postoperative X-ray (D).

sults included Constant, American Shoulder and Elbow Surgeons (ASES), and visual analog scale (VAS) scores collected at the final follow-up visit after surgery. The radiologic evaluation was conducted using follow-up radiographs for evaluating bony union and complications such as delayed union, nonunion, AVN of the humeral head, metal failure, or implant loosening.

\section{RESULTS}

The mean length of follow-up was 26.2 months (range, 12-54 months). The mean Constant, ASES, and VAS scores at the postoperative final visit were 66.7 (range, 43-93), 65.5 (range, 39-92), and 2.2 points (range, $0-4$ ), respectively (Table 1 ). In this study, the sample size was too small $(n=6)$ to conduct statistical analysis. However, we could not analyze or compare the subjective outcomes score according to the patient groups. Two patients who underwent joint-replacement surgeries showed no radiological complications until the last follow-up and their mean Constant, ASES, and VAS scores at the postoperative final visit were $66.5,65$, and 2.5 points, respectively.

Four patients who underwent ORIF achieved bony union of fracture site radiologically and their mean Constant, ASES, and
VAS scores at the postoperative final visit were $65,67.5$, and two points. Among these four patients, AVN of the humeral head was observed at 6 months (patient 1) and 9 months (patient 3) after ORIF surgery, respectively, and penetration of the screws into the joint was observed in these patients. Therefore, the clinical scores in these patients were lower. One individual (patient 1) underwent an additional operation for screw replacement at six months after the initial operation and AVN was observed at final follow-up (Fig. 8). The other patient refused additional surgery and has remained under observation (Fig. 9).

\section{DISCUSSION}

We reviewed six patients with LPFDS in this study. This rare injury often occurs in relatively young patients following high-energy trauma; therefore, a cautious approach to treatment is needed. Previous reports have shown that closed reduction under general anesthesia produces good results in acute cases $[1,9,10]$. However, good results in cases of this locked type of fracturedislocation injury are difficult to achieve by closed methods and there are many factors to consider when performing surgical treatment. 

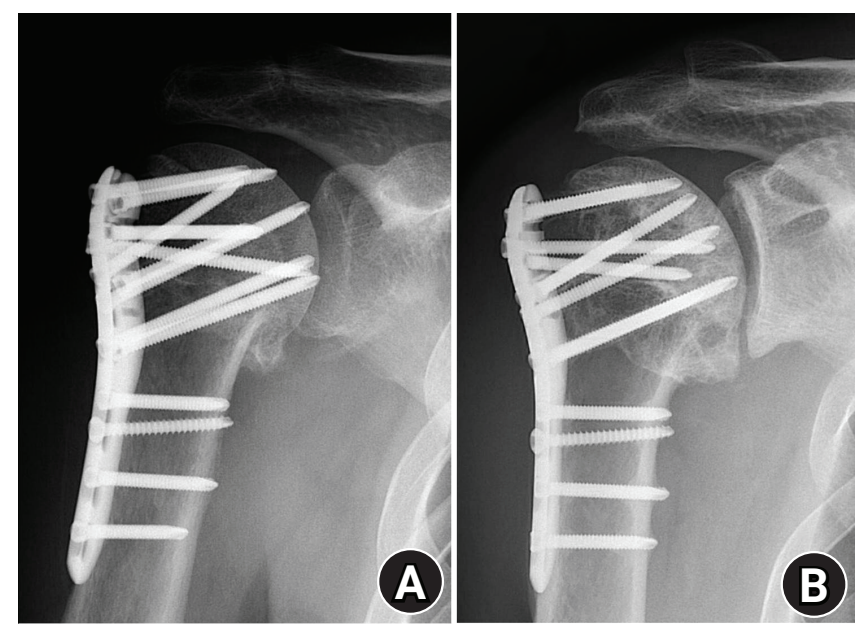

Fig. 8. X-ray of patient 1 showing penetration of screws into the joint 6 months after the initial surgery (A). Screw replacement was performed but avascular necrosis of the humeral head was observed 54 months after the initial surgery (B).

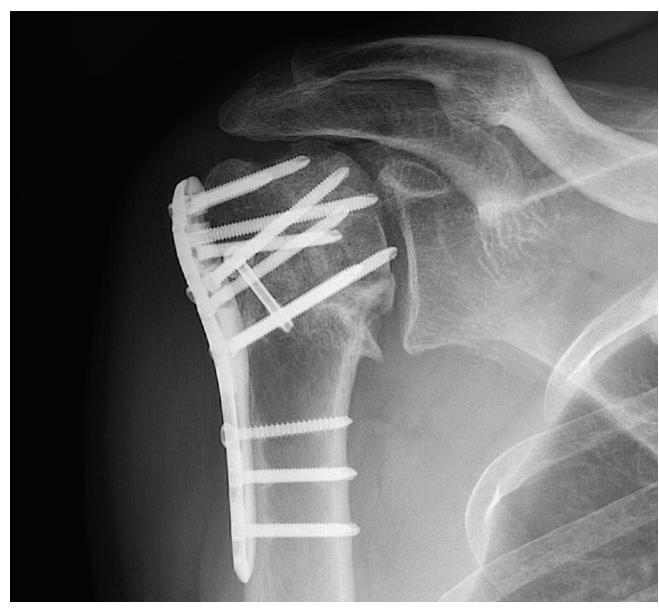

Fig. 9. X-ray of patient 3 at 27 months after the initial surgery. Screw penetration into the joint and avascular necrosis of the humeral head were observed. The patient refused additional surgery and has remained under observation.

Analyzing the pattern of fracture will help with understanding the mechanism of injury. In the present study, all patients were classified as four-part fractures of the Neer classification [3]. As reported by previously published studies $[1,6]$, preoperative three-dimensional computed tomography (3D CT) findings in our series cases show consistent fracture patterns. The fracture occurred in the anatomical neck and articular surface of the humeral head faced posteriorly. Fractures of greater and lesser tuberosity were observed; however, they were nondisplaced or minimally displaced. It can be assumed that the reasons for why this fracture pattern occurred during the series included processes of dislocation and reduction of the posterior dislocation. It can be supposed that the fracture begins at the anterior osteochon- dral lesion of the anatomical neck and then the humeral head becomes locked into the posterior glenoid rim. Next, the fracture was extended posteriorly by reduction force, leading to a complete fracture of the humeral head and, subsequently, the greater tuberosity, the lesser tuberosity, and the shaft. Finally, the humeral head remained in a displaced position, facing posteriorly. This fracture pattern is similar to the principle of opening a bottle cap.

Due to unique characteristics of the fracture, the closed method exhibits difficulty in reducing the locked articular fragments and achieving good results, which usually requires open-reduction surgery. Decisions will be needed on whether to perform osteosynthesis surgery or to perform the joint replacement surgery before or during surgery. Joint-replacement surgery can be considered in the context of chronic or neglected LPFDS, a severe fracture to the articular surface ( $>50 \%$ ), or elderly patients [11]. In the past, hemiarthroplasty or anatomical total shoulder-replacement surgery have often been performed to address this type of injury. Recently, however, reverse total-shoulder arthroplasty (RTSA) surgery has produced more reliable results for both proximal humerus fractures [12] and for this type of injury. In this study, the operation method was chosen mainly according to the patient's age. The average age of the two patients who underwent replacement surgery was 76 years old, while those who underwent osteosynthesis surgery were on average 44 years old. The final clinical results of patients who underwent replacement surgery were similar to those of the patients who underwent osteosynthesis surgery without AVN. The RTSA patient showed better clinical results than the patient who underwent hemiarthroplasty surgery.

In this study, the incidence rate of AVN of the humeral head after ORIF was higher than that in other recent published studies $[6,7]$. However, the number of ORIF procedures $(n=4)$ included in this study was too small to compare with other studies. Although it is difficult to accurately compare the outcomes of this rare injury among studies, the cases in this study included Neer four-part fractures, which are marked by high rates of complications. Further, the use of a deltopectoral approach would have led to longer surgical times with additional soft-tissue damage, while the selection of bone-graft material may have affected the results.

Our retrospective analysis of the patients included herein showed that there were many factors to consider. Once the osteosynthesis surgery is decided, several factors can drive better outcomes. First, surgery should be performed as soon as possible so that it can be completed without swelling or muscle contractures [7]. This will prevent further damage to the soft tissue and bone. Second is the surgical approach. The deltopectoral approach, which has been widely used in shoulder surgery, was adopted in 
all current cases. However, as reported in previous literature, this approach shows some problems when treating this type of injury [13-15]. Specifically, there is a limitation to securing posterior space because this approach is done through the anterior intermuscular plane. The humeral head is locked in the posterior rim of glenoid and it is very difficult to restore the joint surface from the posterior direction. In this case series, the ORIF surgery took longer (average, 128 minutes) than replacement surgery (average, 96 minutes) to perform. Depending on our experience and other authors' reports [7], different approaches may be warranted to facilitate easy and rapid reduction of the humeral head. Although closed, percutaneous, or arthroscopy-assisted techniques have been introduced, we believe that direct identification of the fracture site is a safe and certain way to treat this type of injury. Third is the fixation method. In this study, locking plates (PHILOS plate; Depuy Synthes, Johnson \& Johnson, Oberdorf, Switzerland) were used. Conversely, in the first report of this type of injury, compression screws were used [13] and, later, traditional plates were used. Since it has been reported that it is excellent to use locking plates for proximal humerus fixation in both normal and osteoporotic bone [16,17], this type of injury requires locking plate fixation. Finally, the choice of bone graft is one of the factors that can affect the results because bone defects are often seen in this type of injury. In this study, allograft bone-chip grafting was performed for bone defects found at the site of fracture. Although bony union was achieved in all ORIF cases, osteonecrosis with screw penetration occurred in two of the four cases. It was assumed that the allograft bone chip could serve as filler, but the level of biomechanical support during fracture healing apparently was insufficient. Recently, there have been many reports of excellent results when using fibular allograft in proximal humerus fractures [18]. We believe that such could be considered as one of the options for bone grafting in this type of injury.

There are limitations to this study. Due to the rarity of this injury, only a few patients were included. Young to middle-aged patients underwent surgery to obtain osteosynthesis, while elderly patients underwent replacement surgery; as such, it was difficult to compare each technique. It is believed that due to various surgical treatment methods, the same rehabilitation protocols were not applied in all cases, which could have affected the treatment outcomes. Second, a longer follow-up period would have yielded more reliable rates of complications and prognosis. In particular, the rate of complications such as osteonecrosis and traumatic osteoarthritis could have increased if longer follow-up was designated for the ORIF patients. Finally, a small number of cases made it difficult to complete a meaningful comparison. It is believed that a large multicenter, randomized, controlled study is necessary in the future to analyze various factors affecting the results.

LPFDS is a rare form of injury, with few literature reports available that discuss its management. During diagnosis, a 3D CT scan will help to identify the pattern of injury. When ORIF surgery is applied to young patients, efforts should be made to avoid complications such as osteonecrosis. Making choices regarding parameters such as the surgical approach, implant system, bone-grafting method for shortening the surgical time, and rigid fixation may affect the postoperative surgical outcomes; therefore, these decisions should be made carefully.

\section{ORCID}

Ho Yeon Park Seok Jung Kim Yoo Joon Sur Jae Woong Jung Chae-gwan Kong https://orcid.org/0000-0001-8781-5533 https://orcid.org/0000-0002-9116-8786 https://orcid.org/0000-0003-0090-9970 https://orcid.org/0000-0002-7817-2422 https://orcid.org/0000-0003-4237-4556

\section{REFERENCES}

1. Kowalsky MS, Levine WN. Traumatic posterior glenohumeral dislocation: classification, pathoanatomy, diagnosis, and treatment. Orthop Clin North Am 2008;39:519-33.

2. Neer CS 2nd. Displaced proximal humeral fractures. II. Treatment of three-part and four-part displacement. J Bone Joint Surg Am 1970;52:1090-103.

3. Neer CS 2nd. Displaced proximal humeral fractures. I. Classification and evaluation. J Bone Joint Surg Am 1970;52:1077-89.

4. Sheehan SE, Gaviola G, Sacks A, Gordon R, Shi LL, Smith SE. Traumatic shoulder injuries: a force mechanism analysis of complex injuries to the shoulder girdle and proximal humerus. AJR Am J Roentgenol 2013;201:W409-24.

5. Rouleau DM, Hebert-Davies J. Incidence of associated injury in posterior shoulder dislocation: systematic review of the literature. J Orthop Trauma 2012;26:246-51.

6. Robinson CM, Akhtar A, Mitchell M, Beavis C. Complex posterior fracture-dislocation of the shoulder: epidemiology, injury patterns, and results of operative treatment. J Bone Joint Surg Am 2007;89:1454-66.

7. Branca Vergano L, Landi S, Monesi M. Locked posterior fracture-dislocation of the shoulder. Acta Biomed 2019;90:139-46.

8. Kokkalis ZT, Iliopoulos ID, Antoniou G, Antoniadou T, Mavrogenis AF, Panagiotopoulos E. Posterior shoulder fracture-dislocation: an update with treatment algorithm. Eur J Orthop Surg Traumatol 2017;27:285-94. 
9. Spencer EE Jr, Brems JJ. A simple technique for management of locked posterior shoulder dislocations: report of two cases. J Shoulder Elbow Surg 2005; 14:650-2.

10. Duralde XA, Fogle EF. The success of closed reduction in acute locked posterior fracture-dislocations of the shoulder. J Shoulder Elbow Surg 2006;15:701-6.

11. Cheng SL, Mackay MB, Richards RR. Treatment of locked posterior fracture-dislocations of the shoulder by total shoulder arthroplasty. J Shoulder Elbow Surg 1997;6:11-7.

12. Dillon MT, Prentice HA, Burfeind WE, Chan PH, Navarro RA. The increasing role of reverse total shoulder arthroplasty in the treatment of proximal humerus fractures. Injury 2019;50:67680.

13. Stableforth PG, Sarangi PP. Posterior fracture-dislocation of the shoulder: a superior subacromial approach for open reduction. J Bone Joint Surg Br 1992;74:579-84.

14. Fiorentino G, Cepparulo R, Lunini E, Guardoli A, Berni L, Fon- tanarosa A, et al. Posterior shoulder fracture-dislocation: double approach treatment. Our experience. Acta Biomed 2016;87: 184-90.

15. Saadat W, Monga P. Open reduction and internal fixation of posterior fracture dislocation of the shoulder made easy! J Orthop Case Rep 2017;7:24-6.

16. Jabran A, Peach C, Ren L. Biomechanical analysis of plate systems for proximal humerus fractures: a systematic literature review. Biomed Eng Online 2018;17:47.

17. Laux CJ, Grubhofer F, Werner CM, Simmen HP, Osterhoff G. Current concepts in locking plate fixation of proximal humerus fractures. J Orthop Surg Res 2017;12:137.

18. Zhao L, Qi YM, Yang L, et al. Comparison of the effects of Proximal Humeral Internal Locking System (PHILOS) alone and PHILOS combined with fibular allograft in the treatment of Neer three-or four-part proximal humerus fractures in the elderly. Orthop Surg 2019;11:1003-12. 